\title{
PRODUCCIÓN METALÚRGICA LOCAL Y COEXISTENCIA DE TECNOLOGÍAS METALÚRGICAS EN SAN PEDRO DE ATACAMA DURANTE EL PERÍODO MEDIO (400-1000 DC)
}

\author{
LOCAL METAL PRODUCTION AND COEXISTENCE OF METALLURGICAL \\ TECHNOLOGIES IN SAN PEDRO DE ATACAMA DURING THE MIDDLE \\ PERIOD (400-1000 AD)
}

\author{
Ariadna Cifuentes ${ }^{1}$, Valentina Figueroa ${ }^{2}$ Diego Salazar ${ }^{3}$ y Benoît Mille ${ }^{4}$
}

\begin{abstract}
Durante el período Medio (400-1000 DC) los metales registran un significativo aumento en los cementerios de San Pedro de Atacama, hecho a partir del cual se ha señalado la centralidad de éstos en la estructuración de las relaciones con Tiwanaku. Este trabajo presenta el corpus de metales que circularon en el espacio atacameño durante este período, a través de su identificación tipológica, del reconocimiento de las aleaciones en que fueron manufacturados y de las evidencias productivas. A partir de los resultados, se discute la existencia de una producción metalúrgica local, así como su coexistencia con otras tradiciones tecnológicas metalúrgicas de los Andes Centro Sur, evaluando aspectos relacionados con la producción y uso de los metales.

Palabras claves: producción metalúrgica local, tecnologías metalúrgicas, período Medio, San Pedro de Atacama.
\end{abstract}

During the Middle period (400-1000 AD), metal objects increased significantly in the cemeteries of San Pedro de Atacama, suggesting the centrality of metals in the structuring of relations between local leaders and Tiwanaku. This paper presents the metal objects that circulated in the Atacamenian space during this period through their typological identification, the determination of the alloys in which they were manufactured and their productive evidence. From these results, we discuss the presence of a local metallurgical production and its coexistence with other technological traditions in the South Central Andes. We conclude by evaluating aspects of the social production and use of metals during the Middle Horizon in San Pedro de Atacama.

Key words: Local metal production, metallurgical technologies, Middle Horizon, San Pedro de Atacama.

En la arqueología del Norte Grande, el período Medio (en adelante PM) (ca. 400-1000 DC) se encuentra definido por la aparición del fenómeno de interacción entre poblaciones locales y Tiwanaku (Berenguer y Dauelsberg 1989). En efecto, la evidencia de tal interacción interregional es la aparición de objetos de estilo altiplánico, tales como textiles, cerámicas, tabletas de inhalación y metales, elementos que habrían constituido el soporte material del proceso de consolidación de élites locales "auspiciadas" por Tiwanaku (Berenguer 1998; Berenguer y Dauelsberg 1989; Tarragó 1989; Uribe y Agüero 2001).

Si se compara con el período Formativo (1000 AC-400 DC), los metales en San Pedro de Atacama (en adelante SPA) registran un significativo aumento en las tumbas del PM (Berenguer y Dauelsberg 1989; Llagostera 1996; Núñez 1987; Tamblay 2004). A su vez, se ha argumentado la existencia de una producción metalúrgica local (Llagostera 1996; Núñez 1987, 2006), aunque las evidencias productivas son acotadas, carecen de asignación cronológica clara, y no cuentan con estudios composicionales, desconociéndose qué metales fueron trabajados.

Recientes investigaciones, orientadas a la comprensión de la interacción interregional con Tiwanaku, se han focalizado en el análisis composicional de metales (Lechtman y Macfarlane 2005, 2006; Macfarlane y Lechtman 2015; Maldonado et al. 2010, 2013; Plaza 2015; Salazar et al. 2011). Sus resultados

\footnotetext{
${ }^{1}$ Programa de Doctorado en Antropología UCN-UTA, Universidad Católica del Norte, San Pedro de Atacama, Chile. ariadnacifu@gmail.com

2 Instituto de Arqueología y Antropología, Universidad Católica del Norte, San Pedro de Atacama, Chile. vfigueroa@ucn.cl

${ }^{3}$ Departamento de Antropología, Facultad de Ciencias Sociales, Universidad de Chile, Capitán Ignacio Carrera Pinto $\mathrm{N}^{\circ} 1045$, Santiago, Chile.dsalazar@uchile.cl

${ }^{4}$ Centre de Recherche et de Restauration des Musées de France, 14 Quai F. Mitterrand, 75001 París, France. Préhistoire et Technologie, CNRS UMR7055, Maison Archéologie et Ethnologie René Ginouvès, 21 allée de l'Université, 92023 Nanterre, France. benoit.mille@culture.gouv.fr
} 
han sido coincidentes al desestimar que la metalurgia atacameña sea de carácter local, puesto que en su mayoría se trataría de metales foráneos debido a la presencia de aleaciones no disponibles en la región y/o firmas isotópicas características de materias primas provenientes del Altiplano Boliviano o Noroeste Argentino (en adelante NOA) (Lechtman y Macfarlane 2005, 2006; Macfarlane y Lechtman 2015). No obstante, junto con estos resultados existe un porcentaje de objetos elaborados en cobres no aleados. Esta metalurgia, si bien minoritaria, podría ser de carácter local, puesto que esta composición no ha sido reportada en los principales centros de producción metalúrgica de la época para los Andes Centro Sur (en adelante ACS): Tiwanaku y Aguada (Lechtman 2014). En efecto, en una síntesis reciente, Salazar et al. $(2011 ; 2014)$ plantean la posible coexistencia de tres tradiciones tecnológicas en SPA durante el PM: cobres ternarios de origen altiplánico, bronces con origen en el NOA o Altiplano Boliviano y cobres de alta pureza sin alear, proponiendo a esta última como la expresión de una metalurgia local. No obstante, esta metalurgia local no ha sido definida suficientemente aún, desconociéndose qué tipo de objetos fueron producidos, su relación con la metalurgia altiplánica y otras metalurgias foráneas en las tumbas de SPA, y las implicancias sociales de esta coexistencia, lo que es especialmente relevante considerando la importancia simbólica y política de los metales en los Andes.

\section{Auge Metalúrgico}

Los metales reportados en cementerios del PM de SPA registran un considerable aumento comparado con los hallazgos del período anterior. $\mathrm{Al}$ respecto, destacan los contextos de Larache (Barón 2004; Benavente et al.1986; Tamblay 2004) y Casa Parroquial (Téllez y Murphy 2007), con una notable presencia de objetos de oro. De igual manera, los cementerios de Quitor-5, Quitor-6, Solor-3 y Coyo Oriente registran diversos objetos de base cobre.

El auge metalúrgico del PM es un fenómeno extensible al resto de la región surandina, donde además de un mayor número de objetos, se reporta el manejo de técnicas de manufactura compleja (González 2004) y el uso de aleaciones de base cobre (Lechtman 1996, 2003a, 2003b, 2014). El manejo de aleaciones constituye un verdadero marcador del Horizonte Medio (Lechtman 2003a), sin embargo, no correspondió a un evento único ni homogéneo, coexistiendo distintas tradiciones metalúrgicas, por lo que a partir de la distribución espacial de objetos metálicos, se han observado "fronteras tecnológicas" que serían coincidentes con los límites políticos de la época (Lechtman 2003b, 2014).

En el Altiplano Boliviano las aleaciones $\mathrm{Cu}-\mathrm{As}$ y Cu-As-Ni aparecen sistemáticamente hacia el 600 DC (Lechtman 2003a), registrándose la evidencia más temprana del manejo del $\mathrm{Cu}-\mathrm{Sn}$ durante el Horizonte Medio (Lechtman 2003a, 2003b, 2014). Por su parte, en el NOA los metales fueron producidos en $\mathrm{Cu}-\mathrm{Pb}$ en momentos formativos (Condorhuasi-Alamito) (Pifferetti 1999), y de manera casi contemporánea (Ciénaga) se registra la aleación $\mathrm{Cu}-\mathrm{As}$, y en menor medida $\mathrm{Cu}-\mathrm{Pb}$ y $\mathrm{Cu}-\mathrm{Sn}$. Por su parte, durante el PM se registran con regularidad objetos de $\mathrm{Cu}-\mathrm{Sn}$, aunque en paralelo se producen también artefactos en $\mathrm{Cu}-\mathrm{As}$ (González 2004; Lechtman 2014).

\section{Evidencias metalúrgicas locales}

El área atacameña es una zona esencialmente cuprífera, donde se han reportado actividades extractivas mineras, elementos de ergología minera y evidencias bioantropológicas de mineros desde el período Formativo hasta tiempos coloniales (Bird 1977-1978; Figueroa et al. 2013). Cabe aclarar, sin embargo, que si bien la minería representa la fase inicial de las actividades minero-metalúrgicas, no es posible asumir un consecuente desarrollo metalúrgico a partir de aquélla.

En el área de SPA las evidencias directas de actividades metalúrgicas son escasas y corresponden a escorias metalúrgicas y fragmentos de crisoles o lingoteras. En San Bartolo, Latcham (1936) reporta dos fragmentos de hachas $\mathrm{T}$ junto con escorias; en Coyo y Solor se han recuperado crisoles y lingoteras (Graffam 1995 en Núñez 2006). También Le Paige (1963 en Núñez 2006) señala la presencia de fundiciones en Tchapuchayna; y en Beter, Agüero (2005) ha señalado la existencia de siete fundiciones del PM y/o del Intermedio Tardío (1000-1470 DC), tres de las cuales presentan evidencias formativas. Este conjunto de hallazgos restringidos, inéditos y casi carentes de control cronológico, es insuficiente para demostrar la existencia de una tradición minero-metalúrgica local de raigambre formativa, orientada a la producción de metales, y mucho menos a la exportación de lingotes.

\section{¿Altiplánicos o atacameños?}

Lechtman y Macfarlane (2005, 2006; Macfarlane y Lechtman 2015) analizaron a través de las técnicas de análisis de activación neutrónica y espectrometría de emisión de plasma atómico acoplado inductivamente (ICP-AES), la composición elemental de 36 hachas provenientes de cementerios del PM en SPA. Los 
autores concluyen que en su mayoría estos objetos habrían sido manufacturados en $\mathrm{Cu}-\mathrm{As}-\mathrm{Ni}$, misma aleación que se ha reportado como mayoritaria en Tiwanaku durante los períodos Formativo Tardío 2-Tiwanaku IV hasta Tiwanaku VTemprano (Lechtman 2003a, 2003b, 2014). En menor proporción habrían sido elaboradas en $\mathrm{Cu}-\mathrm{Sn}$, aleación que no habría sido posible desarrollar localmente, dada la inexistencia de menas de estaño en proporciones de importancia. Solo dos hachas mostraron una atípica composición de cobre no aleado. El estudio también evaluó el origen geoquímico de 16 hachas a través de isótopos de plomo, determinándose que habrían sido elaboradas a partir de menas altiplánicas, descartando a SPA como proveedor de las materias primas o productor de tales artefactos, aunque podrían haber sido manufacturadas en la aleación Cu-As-Ni proporcionada por Tiwanaku. Tres hachas podrían provenir desde menas cupríferas próximas a los oasis de SPA (Macfarlane y Lechtman 2015), las cuales desgraciadamente no sabemos si corresponden o no a los objetos elaborados en cobre no aleado.

En esta misma línea, Maldonado et al. (2010, 2013) han efectuado análisis elementales a 17 objetos correspondientes a otras categorías artefactuales a través de la técnica de fluorescencia de rayos X (en adelante FRX). Sus resultados indican que más de la mitad de la muestra corresponde a las aleaciones $\mathrm{Cu}$ As-Ni, mientras que el resto corresponde a la aleación $\mathrm{Cu}-\mathrm{Sn}$, y solo dos objetos a cobres no aleados.

Por su parte, Salazar et al. (2011) determinaron la composición elemental de 11 artefactos metálicos a través de la técnica ICP-AES, siendo sus resultados coincidentescon los estudios anteriores, concluyéndose que en su mayoría se trata de las aleaciones $\mathrm{Cu}$-As$\mathrm{Ni}, \mathrm{Cu}-\mathrm{Sn}$ y $\mathrm{Cu}-\mathrm{Sn}$-As-Ni. Estos resultados sugieren que los objetos fueron importados, dada la ausencia de estaño en la geoquímica local. No obstante, un porcentaje menor corresponde nuevamente a cobres no aleados. A la luz de estos resultados, se plantea en dicho trabajo la hipótesis de manufactura local para los artefactos de cobres no aleados, ya que éstos son atípicos en Tiwanaku y Aguada (Salazar et al. 2011; 2014).

Si bien estos análisis han descartado una metalurgia local mayoritaria, no se ha ahondado suficientemente en la hipótesis sobre una metalurgia de carácter local de cobres no aleados. Por su parte, pese a que han sido tratados aspectos del consumo de metales en SPA en el ámbito funerario (Barón y Serracino 1980; Tamblay 2004), este problema no ha sido abordado a partir de la coexistencia de distintas tradiciones metalúrgicas y sus posibles implicancias sociales.

\section{Material y Método}

De 912 objetos metálicos conservados en el Instituto de Investigaciones Arqueológicas y Museo R.P. Gustavo Le Paige (en adelante IIAM) de SPA, fueron seleccionados aquellos que constituyeran piezas completas o semi-completas, con contexto conocido $\mathrm{y}$ adscritas a cementerios del PM a partir de la presencia de cerámicas y otros artefactos cronológicamente diagnósticos en las tumbas. De manera complementaria, se registraron las evidencias de producción metalúrgica presentes en los cementerios de los oasis.

La muestra seleccionada a partir de los criterios anteriores ascendió a 243 metales, los que fueron recontextualizados a nivel de tumba. Para cada pieza se llevó a cabo un reconocimiento inicial y fichaje individual, el que consideró el relevamiento de dimensiones métricas básicas, descripción morfológica y asignación tipológica, diagnóstico del estado de conservación y fotografía. Fueron sometidas a análisis 26 piezas de composición elemental a través de la técnica de FRX en el Laboratorio de Geoquímica del Departamento de Geología de la Universidad de Chile. Los análisis no se realizaron directamente sobre la superficie de los objetos, sino que se obtuvieron a partir de pequeñas muestras de metal libre de corrosión, extraídas mediante microperforación, asegurando de este modo que los resultados no estuvieran sesgados por los productos de corrosión que cubren los objetos. La Tabla 1 proporciona los resultados de los análisis FRX, mientras que la Tabla 2 y la Figura 1 muestran los resultados obtenidos sobre Materiales de Referencia Certificados analizados bajo el mismo procedimiento que las piezas arqueológicas. Esta información fue complementada con 16 datos composicionales publicados previamente (Lechtman y Macfarlane 2005, 2006; Maldonado et al. 2010; Salazar et al. 2011).

\section{Resultados}

La muestra estudiada fue agrupada en 19 categorías artefactuales correspondientes a cuatro grandes categorías de objetos: armas-emblemas, adornos personales, herramientas y elementos ceremoniales (Figura 2). Bajo el concepto de armasemblemas (sensu Nielsen 2007a) se han reunido aquellos objetos que corresponden a símbolos de poder y autoridad, integrado por hachas, mazas y brazales. Los adornos personales corresponden a objetos de tipo portable utilizados como ornamentos. Por su parte, las herramientas corresponden a objetos utilitarios, incluyendo cinceles y punzones. Por último, los elementos ceremoniales corresponden a 


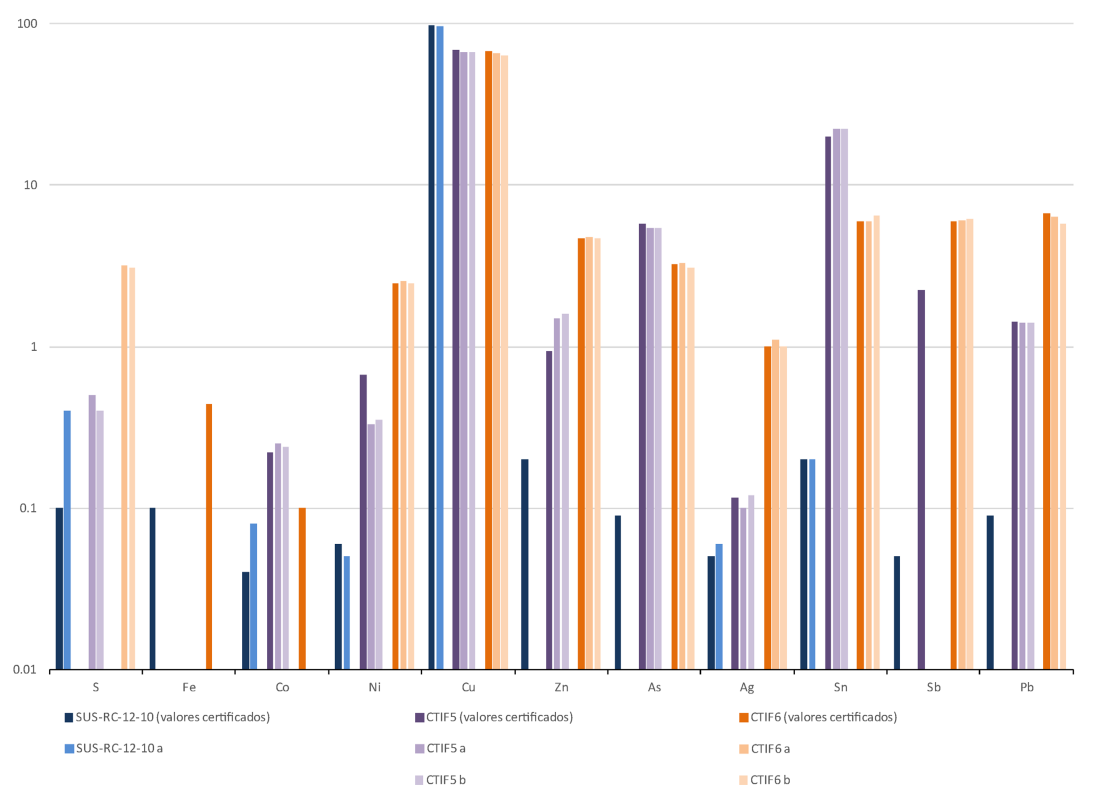

Figura 1. Resultados obtenidos sobre Materiales de Referencia Certificados analizados por FRX de acuerdo al mismo procedimiento que los artefactos arqueológicos.

Results obtained on Certified Reference Materials analysed by XRF according to the same procedure as the archaeological artifacts.

Tabla 1. Composición química elemental de los objetos metálicos analizados por FRX.

Elemental chemical composition of the elemental metal objects analyzed by FRX.

\begin{tabular}{|c|c|c|c|c|c|c|c|c|c|c|c|c|c|c|c|}
\hline $\mathrm{N}^{\circ}$ de Lab. & $\begin{array}{c}\mathrm{N}^{\circ} \mathrm{de} \\
\text { Inventario }\end{array}$ & Cat. Artefactual & Procedencia & $\begin{array}{l}\mathrm{S} \\
\%\end{array}$ & $\begin{array}{l}\mathrm{Cl} \\
\%\end{array}$ & $\begin{array}{l}\mathrm{Fe} \\
\%\end{array}$ & $\begin{array}{l}\text { Co } \\
\%\end{array}$ & $\begin{array}{l}\mathrm{Ni} \\
\%\end{array}$ & $\begin{array}{l}\mathrm{Cu} \\
\%\end{array}$ & $\begin{array}{l}\mathrm{Zn} \\
\%\end{array}$ & $\begin{array}{l}\text { As } \\
\%\end{array}$ & $\begin{array}{l}\mathrm{Ag} \\
\%\end{array}$ & $\begin{array}{l}\text { Sn } \\
\%\end{array}$ & $\begin{array}{l}\mathrm{Sb} \\
\%\end{array}$ & $\begin{array}{l}\mathrm{Pb} \\
\%\end{array}$ \\
\hline PMPC 196 & 3487 & Hacha masiva & Solor-3, T. 487 & 0,06 & n.d. & n.d. & n.d. & n.d. & 94,4 & n.d. & n.d. & n.d. & n.d. & n.d. & n.d. \\
\hline PMPC 187 & 16789 & Brazal & Solor-3, T. 941 & 0,26 & 3,08 & 1,38 & n.d. & 0,09 & 83,7 & n.d. & 0,08 & n.d. & 4,13 & n.d. & n.d. \\
\hline PMPC 192 & $\mathrm{~s} / \mathrm{n}$ & Hacha T & Toconao Or., T. 4576 & 0,24 & n.d. & n.d. & n.d. & 2,79 & 91,9 & n.d. & 2,48 & n.d. & n.d. & n.d. & n.d. \\
\hline PMPC 197 & $\mathrm{~s} / \mathrm{n}$ & Hacha T & Solor-3, T. 455 & 0,69 & 2,64 & n.d. & n.d. & 0,17 & 77,4 & n.d. & 0,1 & n.d. & 6,86 & n.d. & n.d. \\
\hline PMPC 198 & $\mathrm{~s} / \mathrm{n}$ & Hacha T & Solor-3, T. 454-81 & 0,16 & n.d. & n.d. & n.d. & 2,62 & 93,8 & n.d. & 3,24 & n.d. & n.d. & 0,1 & n.d. \\
\hline PMPC 65 & 16472 & Hacha T & Solor-3, T. 913 & 0,32 & n.d. & n.d. & n.d. & 2,39 & 93,7 & n.d. & 3,38 & n.d. & n.d. & 0 & 0,06 \\
\hline PMPC 79 & 16476 & Hacha T & Séquitor Al. Or., T. 1665 & 0,42 & n.d. & n.d. & n.d. & 4,42 & 90,6 & n.d. & 4,03 & n.d. & n.d. & n.d. & n.d. \\
\hline PMPC 287 & 18356 & Hacha T & Quitor-6, T. 2789-92 & 0,1 & n.d. & n.d. & n.d. & 3,94 & 92,7 & n.d. & 3,23 & n.d. & n.d. & n.d. & n.d. \\
\hline PMPC 161 & 16378 & Punzón & Quitor-2, T. 3678-79 & 0,51 & 0,79 & n.d. & n.d. & n.d. & 94,3 & n.d. & n.d. & n.d. & 3,12 & n.d. & n.d. \\
\hline PMPC 201 & 3849 & Cincel & Solcor-3, T. 108 & 0,98 & 1,14 & n.d. & n.d. & 0,15 & 94,3 & n.d. & n.d. & 0,02 & 1,85 & n.d. & n.d. \\
\hline PMPC 24 & 16333 & Punzón & Quitor-6, T. 2426 & 0,23 & 1,33 & 1,19 & n.d. & 0,06 & 90,3 & n.d. & n.d. & n.d. & n.d. & n.d. & n.d. \\
\hline PMPC 121 & 16334 & Punzón & Quitor-6, T. 2427 & 1,07 & 0,92 & n.d. & n.d. & 0,14 & 97,6 & n.d. & n.d. & n.d. & n.d. & n.d. & n.d. \\
\hline PMPC 183 & $\mathrm{~s} / \mathrm{n}$ & Punzón & Quitor-2, T. 3702-04 & 0,12 & n.d. & n.d. & n.d. & n.d. & 89 & n.d. & n.d. & n.d. & n.d. & n.d. & n.d. \\
\hline PMPC 97 & 16310 & Punzón & Coyo Oriente & 0,05 & 1,82 & n.d. & n.d. & 0,07 & 90,8 & n.d. & n.d. & n.d. & 0,11 & n.d. & n.d. \\
\hline PMPC 162 & 16370 & Cincel & Coyo Occ. 10 , rec. 2 , n. 6 & 0,11 & 0,58 & n.d. & n.d. & 1,42 & 96 & n.d. & 0,83 & n.d. & n.d. & n.d. & 0,03 \\
\hline PMPC 106 & 16081 & Punzón & Tchecar Túmulo Sur & 0,6 & n.d. & 1,54 & n.d. & 0,15 & 97,3 & n.d. & 0,09 & n.d. & n.d. & n.d. & 0,01 \\
\hline PMPC 146 & 16788 & Hacha simple & Solcor-3, T. 439 & 0,22 & 1,05 & n.d. & n.d. & 2,66 & 93,4 & n.d. & 2,01 & n.d. & 0,12 & n.d. & n.d. \\
\hline PMPC 207 & 16796 & Maza & Solor-3, T. 486-7 & n.d. & n.d. & n.d. & n.d. & n.d. & 97,2 & n.d. & 0,11 & 0,04 & n.d. & n.d. & n.d. \\
\hline PMPC 199 & $\mathrm{~s} / \mathrm{n}$ & Hacha T & Quitor-5, T. 1994 & 0,13 & n.d. & n.d. & n.d. & 1,81 & 93,6 & n.d. & 3,9 & n.d. & n.d. & n.d. & n.d. \\
\hline PMPC 184 & $\mathrm{~s} / \mathrm{n}$ & Cincel & Quitor-2, T. 3702-04 & 0,23 & n.d. & n.d. & n.d. & n.d. & 99,3 & n.d. & n.d. & n.d. & n.d. & n.d. & 0,03 \\
\hline PMPC 194 & 2395 & Metal aberrante & Solcor-3, T. 64 & 0,28 & 0,67 & n.d. & n.d. & n.d. & 96,9 & n.d. & n.d. & n.d. & n.d. & n.d. & n.d. \\
\hline PMPC 289 & 16521 & Barra no modificada & Coyo & n.d. & n.d. & n.d. & n.d. & n.d. & 99,5 & n.d. & n.d. & 0,04 & n.d. & n.d. & n.d. \\
\hline PMPC 122 & 16340 & Punzón & Quitor-6, T. 2518 & 0,07 & n.d. & n.d. & n.d. & n.d. & 98,5 & n.d. & n.d. & n.d. & 1,12 & n.d. & n.d. \\
\hline PMPC 27 & 16323 & Campanita estrellada & Quitor-6, T. 3619 & 2,38 & 12,8 & n.d. & n.d. & 0,08 & 71,7 & n.d. & n.d. & n.d. & 8,52 & n.d. & n.d. \\
\hline PMPC 243 & 16537 & Pluma cefálica & Quitor-6, T. 2532 & 10,1 & 5,31 & n.d. & n.d. & 3,48 & 67 & n.d. & 2,09 & n.d. & n.d. & 0,2 & n.d. \\
\hline PMPC 229 & $\mathrm{~s} / \mathrm{n}$ & Brazalete & Coyo Oriente, T. 5322 & 0,51 & 10,3 & 1,37 & n.d. & 0,82 & 76,7 & n.d. & 0,75 & n.d. & 7,98 & 0,1 & n.d. \\
\hline
\end{tabular}


Tabla 2. Resultados obtenidos sobre Materiales de Referencia Certificados por FRX analizados de acuerdo al mismo procedimiento que los artefactos arqueológicos.

Results obtained on Certified Reference Materials analysed by XRF according to the same procedure as the archaeological artifacts.

\begin{tabular}{cccccccccccc}
\hline $\mathrm{N}^{\circ}$ análisis & $\mathrm{S}$ & $\mathrm{Fe}$ & $\mathrm{Co}$ & $\mathrm{Ni}$ & $\mathrm{Cu}$ & $\mathrm{Zn}$ & $\mathrm{As}$ & $\mathrm{Ag}$ & $\mathrm{Sn}$ & $\mathrm{Sb}$ & $\mathrm{Pb}$ \\
\hline SUS-RC-12-10 (valores certificados) & 0,1 & 0,1 & 0,04 & 0,06 & 98,5 & 0,2 & 0,09 & 0,05 & 0,2 & 0,05 & 0,09 \\
SUS-RC-12-10 a & 0,4 & 0 & 0,08 & 0,05 & 96,1 & 0 & 0 & 0,06 & 0,2 & 0 & 0 \\
& & & & & & & & & & & \\
& & & & & & & & & & & \\
CTIF5 (valores certificados) & & 0 & 0,22 & 0,67 & 68,5 & 0,94 & 5,75 & 0,117 & 19,9 & 2,23 & 1,42 \\
CTIF5 a & 0,5 & 0 & 0,25 & 0,33 & 66,4 & 1,5 & 5,4 & 0,1 & 22,3 & 0 & 1,4 \\
CTIF5 b & 0,4 & 0 & 0,24 & 0,35 & 66,5 & 1,6 & 5,4 & 0,12 & 22,3 & 0 & 1,4 \\
& & & & & & & & & & & \\
CTIF6 (valores certificados) & & 0,44 & 0,1 & 2,46 & 68 & 4,7 & 3,24 & 1 & 6 & 6 & 6,7 \\
CTIF6 a & 3,2 & 0 & 0 & 2,53 & 65,4 & 4,8 & 3,3 & 1,1 & 6 & 6,1 & 6,4 \\
CTIF6 b & 3,1 & 0 & 0 & 2,47 & 63,7 & 4,7 & 3,1 & 1 & 6,5 & 6,2 & 5,8 \\
\hline
\end{tabular}

objetos del ámbito ritual-ceremonial manufacturados en oro o plata, integrado por vasos-keros y vasosretratos, y tubos para inhalar alucinógenos.

La distribución de los objetos metálicos es heterogénea debido a que los cementerios del período son disímiles en composición y carácter (Figura 3), por lo que la variabilidad distribucional se constata en tres aspectos: (i) abundancia, (ii) diversidad, y (iii) patrones de distribución. En relación al primer aspecto, se observa que en algunos cementerios los metales aparecen en abundancia (Casa Parroquial,
Larache Callejón y Larache Rescate) o relativa abundancia (Solcor-3 y Quitor-6), mientras que en otros constituyen bienes escasos (Quitor-2, Quitor-5, Séquitor Alambrado Oriental y Toconao Oriente). Respecto a la diversidad, en algunos cementerios están representadas muchas de las categorías identificadas (Quitor-6, Solcor-3 y Larache Callejón), mientras otros muestran una aparición restringida de categorías (Quitor-2, Coyo-3, Quitor-5 y Larache Rescate). Por último, se advierten notables diferencias en cuanto a los patrones de distribución de los objetos de oro

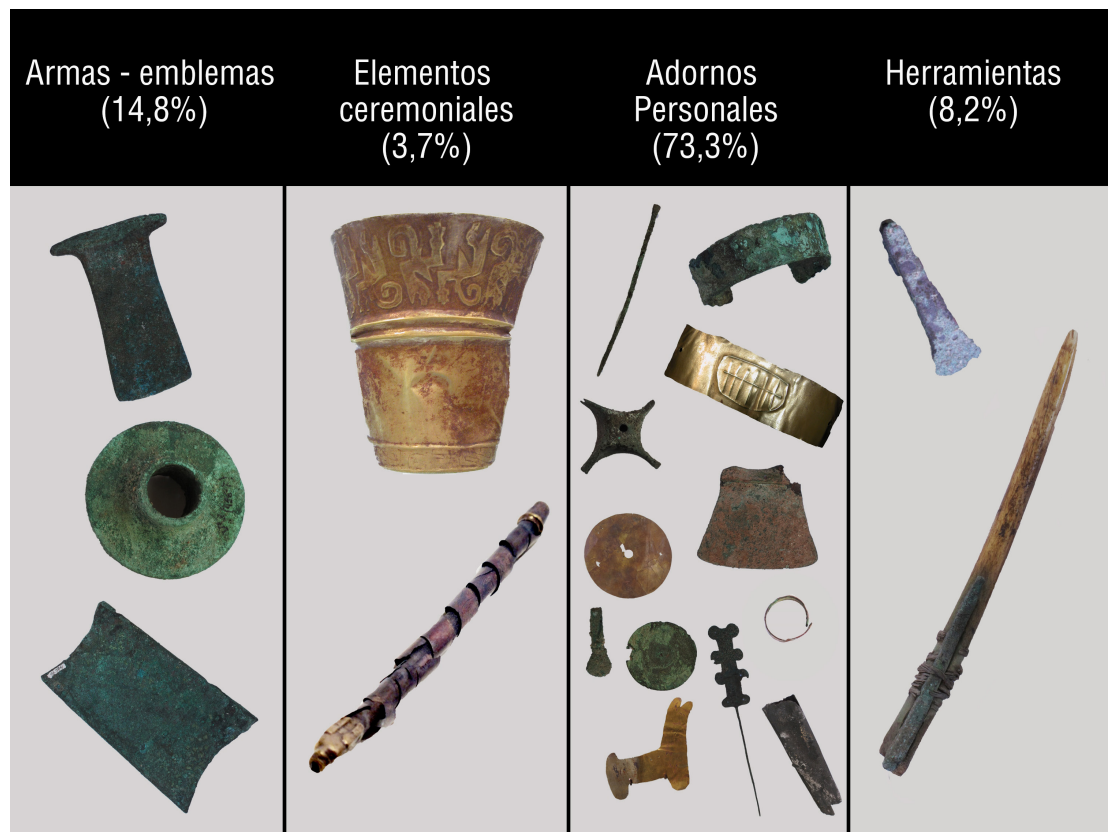

Figura 2. Categorías artefactuales presentes en SPA durante el PM. Artifactual categories present in SPA during the Middle Horizon. 
y plata, presentándose casi exclusivamente en los cementerios centrales de los oasis de SPA (Casa Parroquial, Larache Callejón y Larache Rescate). Por el contrario, las hachas $\mathrm{T}$ muestran una amplia distribución, figurando prácticamente en todos los cementerios, mientras que cinceles y punzones muestran una dispersión acotada, concentrándose en Quitor-2, Quitor-6 y Solcor-3.

\section{Grupos de composición elemental}

Los metales procedentes de los distintos cementerios han sido manufacturados mayoritariamente en cobre o aleaciones de base cobre, no obstante algunos se encuentran elaborados en oro o plata, o aleaciones conbaseenalgunodeestosmetales. A finde determinar las composiciones que caracterizan a este corpus artefactual, definir si éstas tienen relación con las categorías artefactuales identificadas y contribuir a dilucidar la procedencia y alcance geográfico de la circulación de metales para este período, se desarrollaron una serie de análisis de composición elemental (Tabla 1), los que fueron complementados con otros resultados publicados, distinguiéndose los siguientes grupos composicionales (Figura 4):

\section{Cobre-Arsénico-Níquel}

Corresponde al grupo composicional más abundante, dentro del cual se incluye la manufactura de armas-emblemas (14 ejemplares de hachas $\mathrm{T}$ y un hacha simple) y elementos de adorno personal (una pluma cefálica y un disco). También se detectó esta aleación en un cincel proveniente de un nivel estratigráfico de Coyo Occidental, y en una campanita y un disco, para los cuales no se tiene adscripción cronológica segura, por lo que fueron excluidos del análisis.

En términos generales la concentración de arsénico en las piezas bordea entre 3 a $4 \%$, mientras que el níquel se presenta en proporciones cercanas al $3 \%$. Estas proporciones se encuentran dentro de rangos normales de acuerdo a los antecedentes de piezas altiplánicas con esta firma composicional (Lechtman 1996, 2003a, 2003b).

\section{Cobre-Estaño}

Corresponde a los llamados bronces, donde el estaño se presenta en proporciones que bordean entre 1,8 a $8,5 \%$. Se presenta como la segunda composición más abundante, encontrándose manufacturadas en esta aleación armas-emblemas (tres hachas $\mathrm{T}$, un hacha simple y tres brazales), objetos de adorno personal (una campanita) y herramientas (un cincel y dos punzones). También fue identificada en piezas sin adscripción segura, incluyendo un lingote, una campanita y un hacha $\mathrm{T}$.

\section{Cobre-Estaño-Arsénico-Níquel}

Corresponde a una aleación de aparición restringida para la que existen pocos antecedentes. La presencia de estaño oscila entre un 5 a $10 \%$, mientras que el arsénico y níquel figuran en una proporción cercana al $1 \%$. Esta aleación está representada por dos hachas $\mathrm{T}$ y un pequeño brazalete.

\section{Cobres no aleados}

Esta composición se detectó en armasemblemas (dos mazas, un hacha-masiva y un hacha $\mathrm{T}$ ), en herramientas (tres punzones y un cincel) y en elementos de producción metalúrgica (dos lingotillos y un desecho metálico) (Figura 5). Esta composición también fue detectada en un punzón en Quitor-6, un cincel en Coyo Oriente, un topu en Beter (Maldonado et al. 2010, 2013) y un hacha T en Tchecar (Lechtman y Macfarlane 2005, 2006), así como en otros objetos de procedencia superficial, incluyendo una maza en Solor-3 y un punzón en Tchecar. Estos objetos no fueron integrados al análisis por no existir certeza sobre su adscripción cronológica.

Dentro de este grupo hay que distinguir entre cobres de alta pureza y cobres con impurezas. Estas impurezas corresponden a plata, azufre, arsénico y níquel, en concentraciones inferiores a $0,1 \%$, y hierro en concentraciones cercanas a $1 \%$.

Es interesante destacar que objetos tales como las mazas y un hacha-masiva de morfología sin referente en otra área, parecen ser exclusivos de SPA ya que no se los encuentra en regiones vecinas. Coincidentemente, estos objetos fueron elaborados en cobres no aleados, lo que respalda la tesis de una elaboración local. Por su parte, algunas hachas T elaboradas en cobre no aleado podrían sugerir la imitación de morfologías foráneas, idea originalmente planteada por Graffam (en Lechtman 1997). Complementando lo anterior, los datos isotópicos de Lechtman y Macfarlane (2005, 2006; Macfarlane y Lechtman 2015) demostraron que tres de las hachas $\mathrm{T}$ pueden haber sido elaboradas con fuentes cupríferas cercanas a SPA, siendo dos manufacturadas con cobre de la Provincia Isotópica I, coincidente con los depósitos de Chuquicamata, y una elaborada con cobre de la Provincia Isotópica II, coherente con las menas de la mina Chulacao, ubicada al sureste de SPA (Macfarlane y Lechtman 2015:50).

\section{Aleaciones de oro y plata}

En menor proporción se encuentran los objetos manufacturados en oro o plata o aleaciones que 

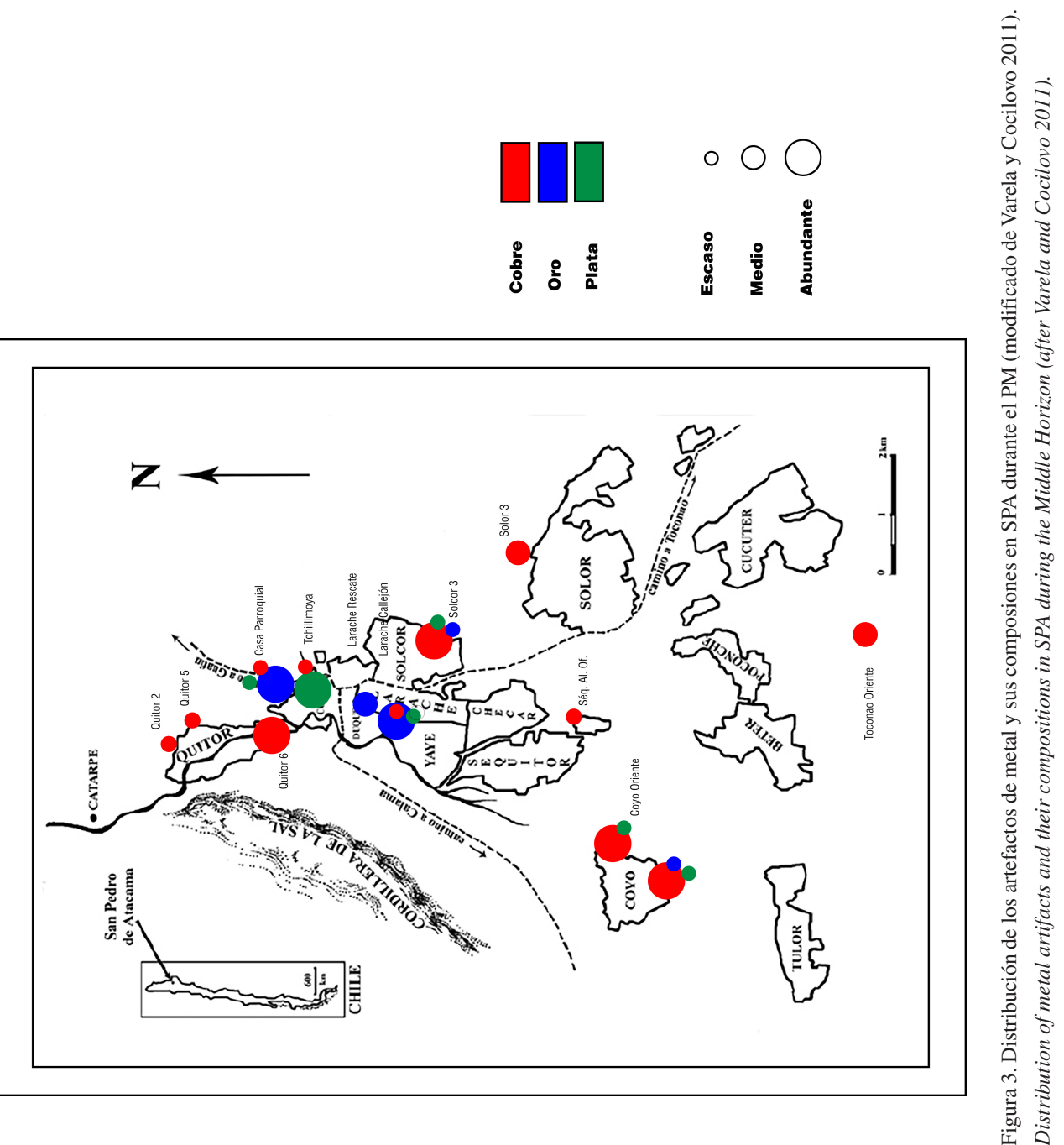


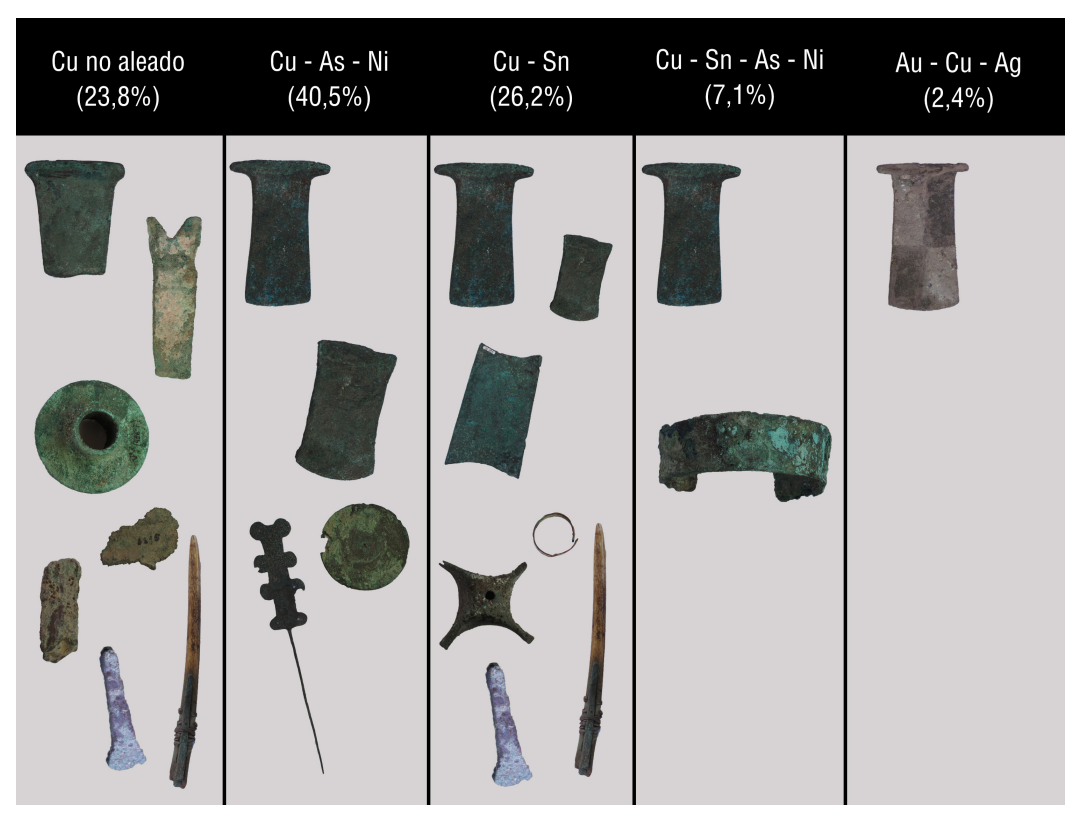

Figura 4. Categorías artefactuales y su representación por grupo composicional. Artifactual categories and their representation by compositional group.

los presentan como elemento base. Corresponden a objetos ceremoniales y de adorno personal, incluyendo diademas, discos, brazaletes y anillos, y excepcionalmente se ha reportado la aleación Au-Cu-Ag para un hacha T (Salazar et al. 2011), la que también ha sido identificada en dos anillos (Téllez y Murphy 2007). Recientes análisis dan cuenta de la complejidad de estas aleaciones, existiendo un gran espectro de variabilidad en los niveles de oro y plata, con una presencia de cobre entre el 0-6\% (Plaza et al. 2015).

\section{Discusión}

La predominancia del grupo composicional $\mathrm{Cu}$ As-Ni en los objetos metálicos de los cementerios del PM de SPA sugiere que en su mayoría los metales presentes en estos oasis circumpuneños correspondieron a objetos importados desde el Altiplano Boliviano, aun cuando no pueda precisarse el área específica de fabricación de estos objetos. La aleación $\mathrm{Cu}-\mathrm{Sn}$ representa una producción foránea. En el Altiplano Boliviano, si bien se populariza a partir de Tiwanaku V, su producción se presentaría en momentos previos en Lukurmata (Lechtman 2003a), pero también aparece tempranamente en el NOA en algunos contextos Ciénaga, registrándose con regularidad durante el período de Integración Regional (400-900 DC) (González 2004). Por su parte, la aleación $\mathrm{Cu}-\mathrm{Sn}-\mathrm{As}-\mathrm{Ni}$, aunque poco conocida, ha sido reportada en Tiwanaku (Lechtman 2003a), por lo que debiera ser una variante del $\mathrm{Cu}-\mathrm{As}-\mathrm{Ni}$. Finalmente, las aleaciones de oro o plata también serían exógenas (Plaza et al. 2015). En consecuencia, debería descartarse la hipótesis de que Tiwanaku incentivó el desarrollo de una producción metalúrgica local excedentaria para abastecer de metales al centro altiplánico (Berenguer y Dauelsberg 1989; Bird 1977-1978; Núñez 1987, 2006). No obstante, estos resultados no invalidan la posibilidad de que se haya desarrollado una producción metalúrgica local.

En efecto, los datos tipológicos permiten observar que dentro del corpus de objetos metálicos encontrados en las tumbas del PM de SPA destacan una serie de objetos comunes en los ACS, pero también hay un grupo de objetos que solo se presentan en SPA. Estos objetos, así como otras categorías artefactuales que si se representan a nivel regional, fueron elaborados en cobres no aleados. Estos datos abren la posibilidad de que dicho grupo composicional corresponda a una metalurgia de carácter local. Sin embargo, estos análisis por sí mismos son insuficientes para demostrar la hipótesis. Solo la identificación de desechos metalúrgicos in situ constituye evidencia inequívoca de la existencia de actividades metalúrgicas locales.

En este sentido, si bien en los oasis atacameños no se han encontrado talleres metalúrgicos, existen evidencias productivas en Solor-3, Solor-4, Séquitor 

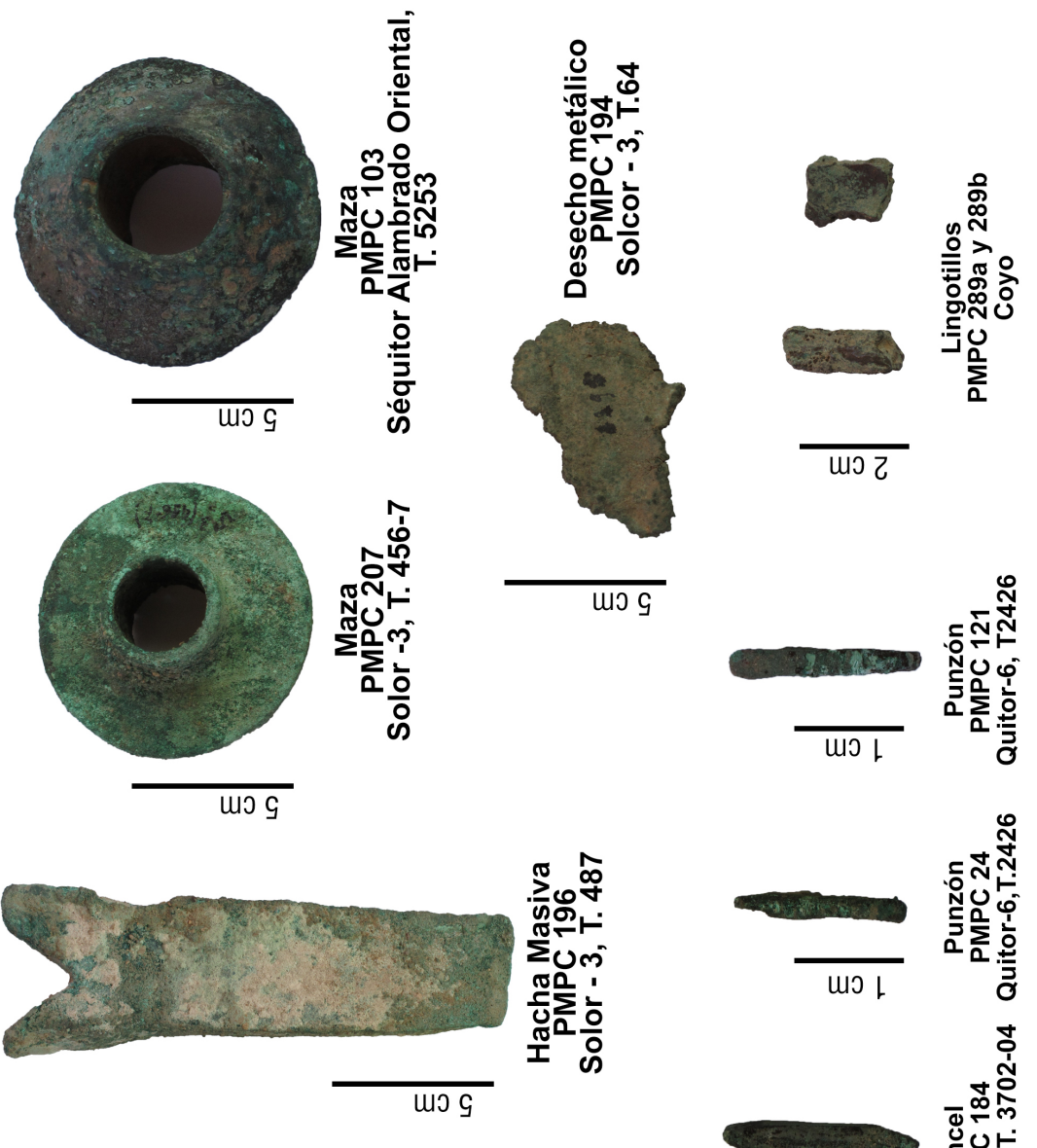

离
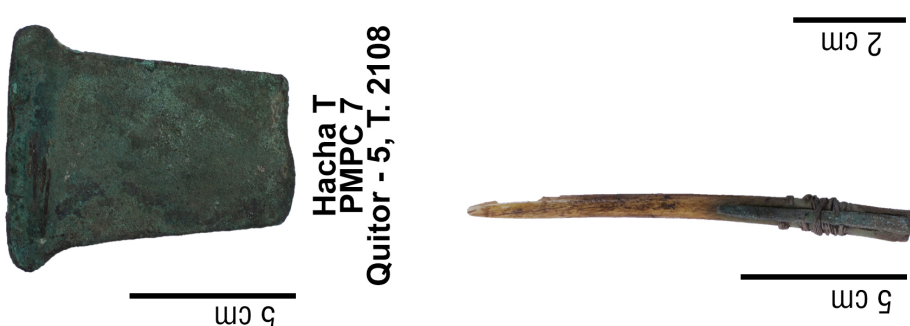
Alambrado Oriental, y los ayllus Coyo y Beter, que confirman el desarrollo de actividades metalúrgicas locales (Figura 6). Dos lingotillos procedentes de la superficie de Coyo y un desecho metálico procedente de una tumba de Solcor-3, fueron sometidos a análisis de composición elemental. Sus resultados indicaron que éstos corresponden a cobres no aleados, lo cual es muy interesante, ya que restringe a esta composición el conjunto de evidencias productivas caracterizadas composicionalmente, nuevamente en concordancia con lo sugerido por los análisis de objetos terminados.

\section{Metales locales en SPA}

El área atacameña posee abundantes riquezas cupríferas con gran potencial para el desarrollo de actividades minero-metalúrgicas (p.ej. San Bartolo, Chulacao, Chuquicamata, Conchi, El Abra, Caspana, Tuina y Peine), con antecedentes de operaciones mineras durante el PM en Chuquicamata (Bird 19771978), El Abra (Salazar et al. 2010) y El Salvador (Westfall y González 2010), y presencia de martillos mineros en Coyo Oriente (Figueroa et al. 2013; Le Paige 1972-1973) y Coyo-3 (Costa y Llagostera 1994). Como hemos señalado, esta tradición minera no constituye por sí sola evidencia de producción metalúrgica, aunque este elemento conjugado con evidencias directas e indirectas apoyan la hipótesis de una producción local de objetos en el PM, a partir de recursos cupríferos atacameños. A continuación sintetizamos los argumentos que permiten respaldar esta hipótesis.

En primer lugar, existen objetos cuya tipología no ha sido reportada en otras áreas de los ACS y que, por lo tanto, parecen haber sido elaborados de manera exclusiva en SPA. Dentro de éstos cabe consignar un hacha-masiva ${ }^{1}$ y las mazas. Éstas últimas presentan una morfología distinta a sus homólogas peruanas (Mayer 1998), asemejándose solamente a ejemplares bolivianos descontextualizados (Mayer 1994), y siendo morfológicamente similares a las mazas de piedra características del Formativo Tardío atacameño (Llagostera 2006).

En segundo lugar, existe correspondencia entre los artefactos de morfología desconocida en otras regiones y su composición a partir de cobres no aleados, la que además es muy poco frecuente fuera del área atacameña ${ }^{2}$.

En tercer lugar, existen evidencias productivas que demuestran que en los oasis de SPA sí se desarrollaron actividades metalúrgicas, quizás incluso desde el Formativo Tardío (Agüero 2005). Los elementos productivos incluyen tanto desechos vinculados a la fundición, además de productos metálicos semi-terminados (lingotillos). Cabe destacar que los elementos productivos y desechos metálicos analizados en este trabajo también corresponden a cobres no aleados, lo que refuerza la idea de que es este tipo de metalurgia la que se está desarrollando localmente.

En cuarto lugar, y complementando lo anterior, resulta interesante comentar la presencia de una inhumación (tumba 64, Solcor-3) 3 con ofrendas relacionadas con la producción metalúrgica: un conjunto de prills ${ }^{4}$, una pequeña lámina perforada de plata y un desecho metálico. El análisis de composición practicado a esta última ha indicado que se trata de un cobre no aleado.

Esta confluencia de distintas líneas de evidencia demuestra que efectivamente se produjo una metalurgia local de cobres no aleados en SPA durante el PM, aun cuando ésta fue de escala reducida y cuyos objetos fueron minoritarios $(23,8 \%)$ en comparación con los metales importados $(76,2 \%)$.

\section{Metales foráneos en SPA}

Como ya se ha señalado a partir de los resultados composicionales, el repertorio de metales identificados en SPA corresponde mayoritariamente a objetos foráneos. De la misma manera, a partir de la comparación tipológica del corpus metálico atacameño con regiones vecinas, se observa que se trata de tipos artefactuales compartidos en los ACS. No obstante, ciertos tipos metálicos parecen ser específicos de regiones particulares, lo cual permite precisar probables áreas de proveniencia para éstos.

\section{Altiplano Boliviano}

Esta región presenta una serie de tipos artefactuales compartidos con el norte de Chile, NOA y Perú, tales como hachas, mazas, brazales, cuchillos-placa, anillos, alfileres, discos, cinceles y punzones. Quizás el único artefacto que restringe su distribución a esta área son las grapas arquitectónicas, hecho que no sorprende dada la ausencia de arquitectura Tiwanaku en Chile y el NOA.

La aleación Cu-As-Ni corresponde a la firma altiplánica por excelencia, particularmente porque se encuentra espacial y temporalmente circunscrita a la región altiplánica durante el PM (Macfarlane y Lechtman 2015). Se hace presente desde el siglo II en el área de influencia Tiwanaku, siendo característica de los metales más tempranos distribuidos por esta órbita. Junto a esta aleación, también se registran artefactos manufacturados en $\mathrm{Cu}-\mathrm{Sn}$, composición que fue reemplazando progresivamente al $\mathrm{Cu}-\mathrm{As}$ Ni hacia Tiwanaku V (800-1000 DC) (Lechtman 


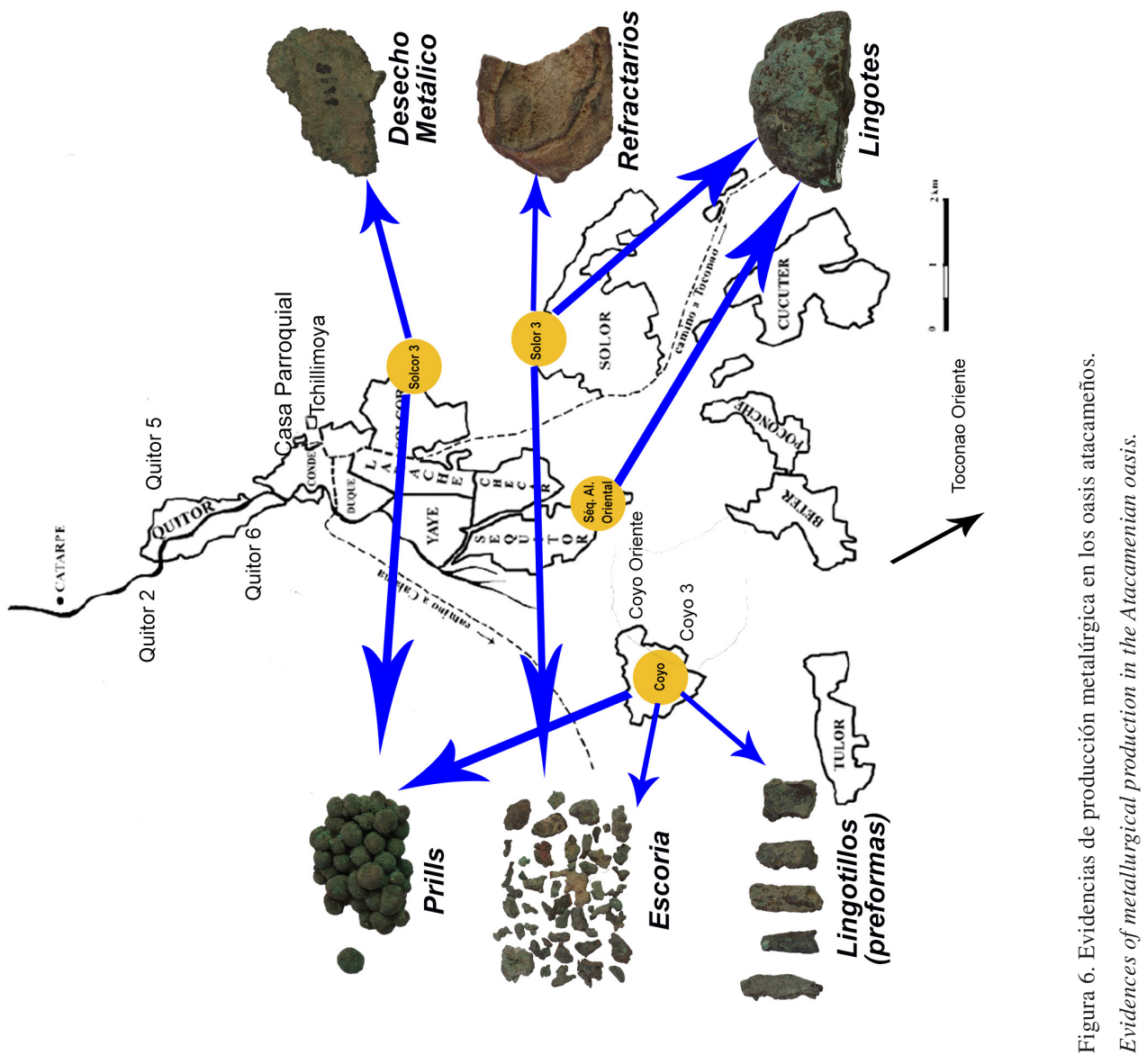


1996, 2003a, 2003b, 2014). Una tercera aleación que podría tener su origen en esta región es la aleación $\mathrm{Cu}-\mathrm{Sn}$-As-Ni. Finalmente, cabe hacer mención a la aleación $\mathrm{Cu}-\mathrm{As}$, la que aparece sistemáticamente hacia el $600 \mathrm{DC}$, pero que no cuenta con referentes en los cementerios atacameños del período, aunque sí se ha reportado en algunos objetos sin información contextual confiable ${ }^{5}$. El centro de producción de esta tradición es desconocido aún, y solo es posible señalar que probablemente haya sido Tiwanaku la entidad encargada de la redistribución de estos metales.

\section{NOA}

Para el PM esta área está definida principalmente por la metalurgia Aguada. Se observan ciertos tipos compartidos de forma exclusiva con el norte de Chile, tales como brazaletes delgados, discos de cobre y campanitas estrelladas, todos objetos escasamente documentados en el Altiplano Boliviano. No obstante, los datos actuales parecen indicar que pocos metales atacameños provienen de Aguada, debido a que los análisis composicionales en objetos morfológicamente semejantes muestran aleaciones totalmente distintas, o patrones de impurezas diferentes. Por otra parte, una cantidad importante de objetos propios del área Aguada (zona valliserrana meridional) no ingresan a los oasis atacameños, tales como hachas $\mathrm{T}$ con motivo felino, hachas-cetros, discos y placas Aguada. Adicionalmente, las hachas T difieren de las atacameñas por su mayor volumen (Ambrosetti 2011 [1904]:66-67).

Dado que prácticamente no hay metales propiamente Aguada, los objetos de $\mathrm{Cu}-\mathrm{Sn}$ reportados en SPA pareciesen venir desde el Altiplano Boliviano o bien desde una tercera zona de producción aún no definida. Estos metales son morfológicamente semejantes a ciertos objetos de $\mathrm{Cu}-\mathrm{Sn}$ de SPA, particularmente hachas T, brazales, campanitas, cinceles y punzones. Pero estos objetos son también idénticos a los del Altiplano Boliviano, por lo que bien pudieron llegar al NOA desde el altiplano, siendo imposible por el momento precisar mejor esta situación. Sin duda, este problema necesita seguir siendo explorado en la medida en que se profundice el estudio de metales de base cobre en el área valliserrana septentrional, para la cual se conocen casi exclusivamente objetos de oro y plata (González 2004:182).

\section{Norte Semiárido 6}

Esta región si bien comparte tipos artefactuales con SPA, tales como anillos, brazales, brazaletes, campanitas, cinceles, cuchillos-placa, diademas, discos, pinzas, punzones y vasos (Corral 2009), otros como mazas, alfileres, orejeras y plumas cefálicas, hachas $\mathrm{T}$ y hachas simples están ausentes, quedando en evidencia el límite que constituyeron los oasis atacameños para la dispersión de estos elementos hacia el sur. A su vez, los aros, populares en contextos Ánimas, están ausentes en el área atacameña. No obstante, en el Norte Semiárido es posible distinguir objetos metálicos comunes tanto a la porción valliserrana meridional como septentrional.

Los datos anteriores permiten observar un panorama general de las metalurgias en los ACS para el PM, el que se expresa en tres situaciones particulares. En primer lugar, SPA y el área valliserrana septentrional habrían constituido el límite sur de un área de dispersión de metales independiente y distinta de la tradición Aguada. Estos datos son coherentes con la diferenciación observada a partir de la distribución de otros elementos de la cultura material en el área valliserrana, puesto que durante el período de Integración Regional, el área valliserrana septentrional parece no haberse relacionado con Aguada y manteniendo en cambio contacto con regiones altiplánicas con influencia Tiwanaku, la región loína y SPA (Tarragó 1999), definiéndose una esfera de interacción particular. $\mathrm{Al}$ respecto es factible pensar que vía SPA podrían haberse establecido los vínculos del núcleo altiplánico con la región valliserrana septentrional, ya que particularmente los tipos metálicos compartidos con esta área corresponden a elementos que son comunes también al Altiplano Boliviano. En segundo lugar, dado que los metales correspondientes a símbolos de estatus no vienen desde Aguada sino que desde Tiwanaku, es posible afirmar que este último habría jugado un rol preponderante en la configuración de las relaciones de desigualdad social en SPA durante el PM, tal como ha sido sugerido por otros investigadores (Llagostera 1996; Salazar et al. 2014). En tercer lugar, la tradición altiplánica y la tradición Aguada se encontrarían juntas en el Norte Semiárido, aunque con predominancia de metales Aguada.

Evaluación aparte merecen los objetos ceremoniales de exclusiva manufactura en oro y plata. Los excepcionales contextos de Larache Callejón, Larache Rescate y Casa Parroquial parecen estar emparentados con otros contextos auríferos registrados en Isla Pariti (Korpisaari et al. 2012), Palacio Putuni (Kolata 1993) y en el "Tesoro de San Sebastián" en Cochabamba (Money 1991), así como también con contextos Isla y Yavi de El Volcán (Gatto 1946 en González 2004:183), Río Doncellas (Rolandi 1974) y la Tumba 11 de Isla de Tilcara (Tarragó et al. 2010), aun cuando estudios recientes han demostrado que los contextos Isla corresponden principalmente a los primeros siglos del segundo milenio DC (Nielsen 2007b). No obstante, la contemporaneidad 
de alfarerías Isla con elementos de la Fase Coyo de SPA (700-1000 DC) parece bien documentada (Tarragó 1989), y existen fechados de contextos Isla de fines del primer milenio. Considerando estos antecedentes, así como los fechados más recientes en SPA que sugieren que la alfarería negro pulido y los contextos con influencia Tiwanaku se mantuvieron en SPA hasta finales del primer milenio e incluso hasta inicios del segundo milenio DC (Hubbe y Torres-Rouff 2013; Richardin et al. 2015; Stovel 2013), es posible postular que los objetos de oro y plata de SPA y la región valliserrana septentrional podrían corresponder a momentos tardíos dentro del $\mathrm{PM}$, muy seguramente en torno a la transición entre el primer y segundo milenios DC, coincidiendo con la parte final de la influencia Tiwanaku en los ACS. Por su parte, en Aguada este tipo de evidencias es prácticamente inexistente (González 2004).

Respecto al centro productor y redistribuidor de estos objetos, si bien no existen datos concluyentes, parecen haber sido elaborados en el Altiplano Boliviano, no solo porque es donde este tipo de hallazgos se registran en mayor cantidad, sino también porque morfologías únicas como los vasos-keros y vasos-retratos, parecen haber tenido referente directo en aquellos elaborados en cerámica y madera.

\section{Consideraciones Finales}

Los metales presentes en SPA para el PM corresponden a tipos artefactuales que circulan intensamente en el espacio de los ACS, pero que lo hacen en distintas órbitas. Se trata de objetos foráneos manufacturados en $\mathrm{Cu}-\mathrm{As}-\mathrm{Ni}$ y $\mathrm{Cu}-\mathrm{Sn}$ correspondientes a armas-emblemas, adornos personales y cinceles y punzones. Excepcionalmente, también se reportan objetos vinculados al ritual y algunos adornos personales, los que se encuentran manufacturados en oro y plata, y que tendrían procedencia exógena. Estos objetos procederían en su mayoría desde el Altiplano Boliviano y habrían llegado a SPA como parte de la interacción con Tiwanaku. Algunos de éstos, también podrían proceder de algún punto del NOA, posiblemente ubicado entre la frontera con Bolivia y los valles calchaquíes, por lo que SPA parece constituir junto con la región valliserrana septentrional un área de confluencia común de circulación de metales con origen en el Altiplano Boliviano.

La predominancia de la aleación $\mathrm{Cu}-\mathrm{As}-\mathrm{Ni}$ sugiere que mayoritariamente correspondieron a objetos procedentes desde el altiplano, posiblemente redistribuidos desde Tiwanaku (fase IV). Asimismo, se identificó una presencia relevante de la aleación $\mathrm{Cu}-\mathrm{Sn}$ en objetos que pudieron proceder desde
Tiwanaku (fase V), aun cuando no puede descartarse un origen en la región septentrional del NOA.

Lo anterior permite proponer una esfera de interacción metalúrgica circumpuneña-vallunaaltiplánica (Figura 7), distinta del área de distribución Aguada que incluiría el Norte Semiárido. Estas particularidades observadas a partir de las áreas de distribución de objetos de metal, permiten plantear la constitución de vínculos políticos entre Tiwanaku, la región valliserrana septentrional y SPA, que encuentran correlato con otras evidencias materiales (Tarragó 1977, 1994).

Junto a estos metales exógenos, existiría una metalurgia local de baja escala en SPA, correspondiente a la producción de metales elaborados a partir de cobres no aleados, orientada a la elaboración de cinceles y punzones, y en menor medida, algunos emblemas de poder, los cuales no lograron una alta destreza técnica ${ }^{7}$. La fabricación de herramientas parece continuar una tradición local (Figueroa et al. 2010), que podría haberse incrementado en el PM para proveer de herramientas a los artesanos de la madera u otro tipo de artesano especializado de la época. A su vez, algunos líderes locales habrían aprovechado el saber técnico de los metalurgistas atacameños, incentivando la producción de algunos emblemas de autoridad, ya sea imitando los provenientes de Tiwanaku (hachas T elaboradas en cobres no aleados), o bien oponiéndose a ellos al enfatizar formas locales, tanto novedosas (hacha-masiva) como tradicionales (mazas) (Salazar et al. 2014).

El contexto sociohistórico en el cual se desarrolla esta metalurgia local en conjunción con el consumo de metalurgias foráneas, corresponde a una época de fortalecimiento de las estructuras de poder en el escenario de intensos circuitos caravaneros (Llagostera 1996; Núñez y Dillehay 1995 [1979]). Así, las metalurgias que convivieron en el espacio funerario de SPA, delinearon varios niveles jerárquicos entre los oasis y al interior de estos mismos, lo cual demostraría que durante el PM hubo una organización social compleja, distinguiéndose niveles de jerarquía creciente en los contextos funerarios (Salazar et al. 2014; Tamblay 2004; Uribe et al. 2016). En parte, estos distintos niveles se vincularon con la creciente especialización artesanal, así como con los vínculos sociales extrarregionales que cada grupo social definió y logró concretar. Se trató ante todo de un proceso dinámico, no exento de la agencia activa de líderes que buscaron representar y consolidar su autoridad mediante distintas estrategias de acceso a bienes de prestigio (Llagostera 1996), pero también incentivando la producción local, imitando los bienes importados o bien diferenciándose abiertamente con los portadores de aquellos al resaltar la tradición local. 


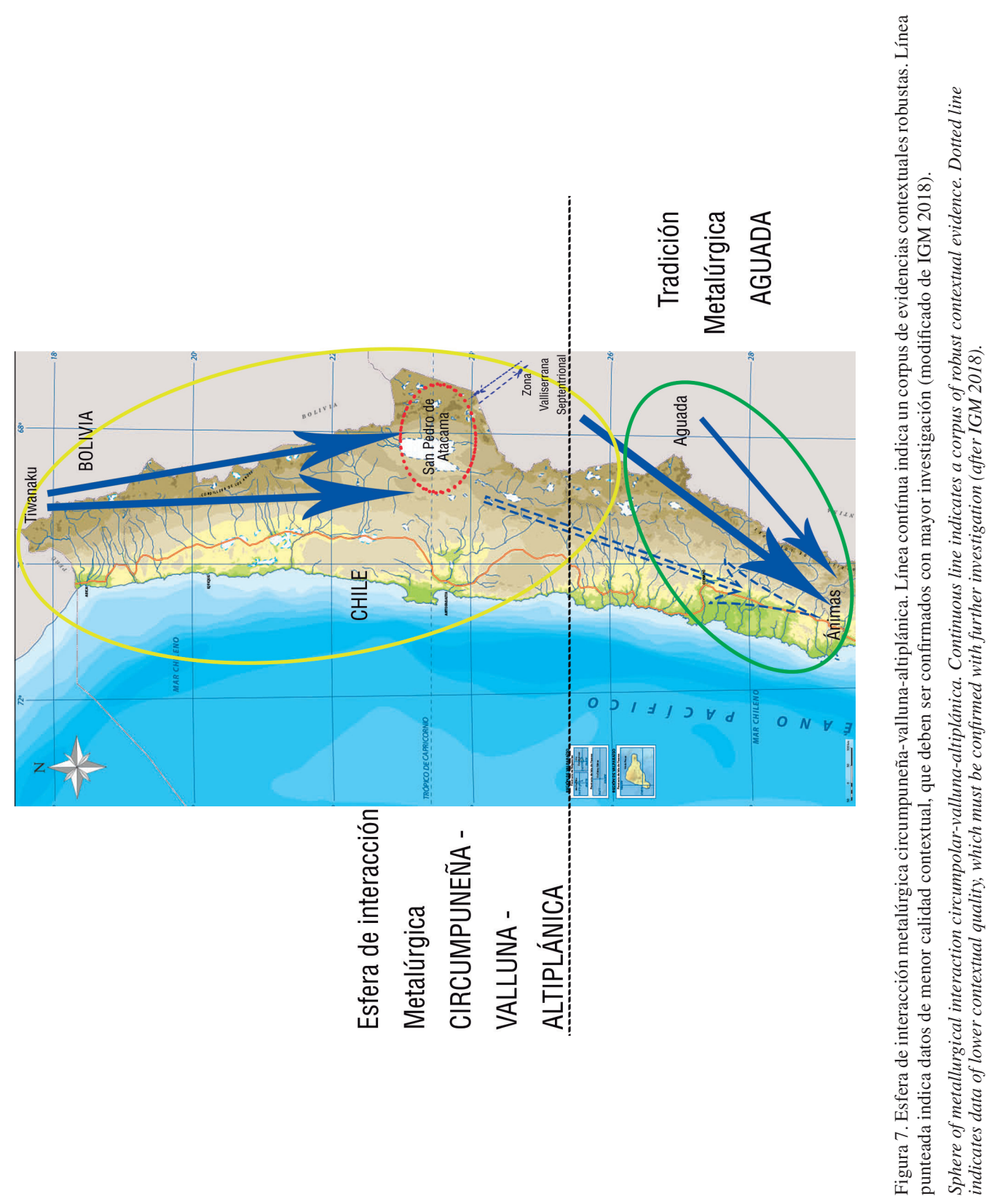


Agradecimientos: Esta investigación ha sido financiada por el Programa de Investigación Asociativa Anillos en Ciencia y Tecnología ACT-96 (http:// www.cienciaymemoria.cl). Los autores agradecen al personal de la Unidad de Colecciones y Conservación
IIAM, a los proyectos ECOS-Conicyt C11H02 y Fondecyt 11130651, a Christian Nievas, encargado del Laboratorio de Geoquímica del Depto. de Geología (Universidad de Chile), y a los evaluadores anónimos de este trabajo, por sus enriquecedores comentarios.

\section{Referencias Citadas}

Agüero, C. 2005. Aproximación al asentamiento humano temprano en los oasis de San Pedro de Atacama. Estudios Atacameños 30:29-60.

Ambrosetti, J. 2011 [1904]. El Bronce en la Región Calchaquí. Facultad de Filosofía y Letras de la Universidad de Buenos Aires, Buenos Aires.

Barón, A.M. 2004. Excavación del Cementerio Larache, Conde Duque en San Pedro de Atacama. En Tiwanaku. Aproximaciones a sus Contextos Históricos y Sociales, compilado por M. Rivera y A. Kolata, pp. 67-97. Universidad Bolivariana, Santiago.

Barón, A.M. y G. Serracino 1980. Rol social de los metales en San Pedro de Atacama. Actas VI Congreso Nacional de Arqueología Argentina, pp.1-12. Jujuy.

Benavente, M.A., C. Massone y C. Thomas 1986. Larrache, evidencias atípicas. ¿Tiahuanaco en San Pedro de Atacama? Chungara 16-17:67-73.

Berenguer, J. 1998. La iconografía del poder en Tiwanaku y su rol en la integración de zonas de frontera. Boletín del Museo Chileno de Arte Precolombino 7:19-38.

Berenguer, J. y P. Dauelsberg 1989. El Norte Grande en la órbita de Tiwanaku (400 a 1.200 d.C.). En Prehistoria. Desde sus Orígenes hasta los Albores de la Conquista, editado por J. Hidalgo, V. Schiappacasse, H. Niemeyer, C. Aldunate e I. Solimano, pp. 129180. Editorial Andrés Bello, Santiago.

Bird, J.1977-1978. El hombre de cobre. Un minero prehistórico del Norte de Chile y sus herramientas. Boletín del Museo Arqueológico de La Serena 16:77-106.

Corral, M.I. 2009. Caracterizando los Objetos Metálicos del Complejo Cultural Ánimas, Norte Semiárido de Chile. Memoria para optar al grado de Arqueólogo. UISEK. Santiago.

Costa, M.A. y A. Llagostera 1994. Coyo-3: Momentos finales del Período Medio en San Pedro de Atacama. Estudios Atacameños 11:73-107.

Figueroa, V., I. Montero y S. Rovira 2010. Estudio tecnológico de objetos de cobre procedentes de Cerro Turquesa (San José del Abra, II Región). Actas del XVII Congreso Nacional de Arqueología Chilena, pp. 1135-1147. Ediciones Kultrún, Valdivia.

Figueroa, V., D. Salazar, H. Salinas, P. Núñez-Regueiro y G. Manríquez 2013. Prehispanic mining ergology of northern Chile: An archaeological perspective. Chungara Revista de Antropología Chilena 45 (1):61-81.

González, L. 2004. Bronces sin Nombre. La Metalurgia Prehispánica en el Noroeste Argentino. Ediciones Fundación Ceppa, Buenos Aires.

Gordillo, I. y H. Buono 2007. Metalurgia prehispánica en el sitio de La Rinconada. (Dpto. Ambato, Catamarca). En La Metalurgia en la América Antigua. Teoría, Arqueología, Simbología y Tecnología de los Metales Prehispánicos, editado por R. Lleras, pp. 421-438. Fundación de Investigaciones Arqueológicas Nacionales, Banco de la República e IFEA, Bogotá.

Instituto Geográfico Militar (IGM) 2018. Mapa de Chile Físico. https://www.igm.cl/div/MAPAIGM/CHILE\%20COMPLETO/ MAPA_DE_CHILE_FISICO_150DPI.jpg (22 de junio de 2018).

Kolata, A. 1993. The Tiwanaku: Portrait of an Andean Civilization. Blackwell, Oxford.

Korpisaari, A., J. Sagárnaga, J. Villanueva y T. Patiño 2012. Los depósitos de ofrendas tiwanakotas de la Isla Pariti, Lago Titicaca, Bolivia. Chungara Revista de Antropología Chilena 44 (2):247-267.

Latcham, R. 1936. Metalurgia Atacameña. Boletín del Museo Nacional 15:107-151.

Lechtman, H. 1996. El bronce y el Horizonte Medio. Boletín del Museo del Oro 41:3-25.

Lechtman, H. 1997. El bronce arsenical y el Horizonte Medio. En Arqueología, Antropología e Historia de los Andes: Homenaje a María Rostorowski, editado por R. Varón y J. Flores, pp. 153-186. IEP, Lima.

Lechtman, H. 2003a. Bronce Metallurgy in the Lake Titicaca Basin: a preliminary assessment. En Tiwanaku and its hinterland. Urban and Rural Archaeology, editado por A. Kolata, pp. 404434. Smithsonian Institution Press, Washington D.C.

Lechtman, H. 2003b. Middle Horizon Bronze: Centers and Outliers. En Patterns and Process. A Festschrift in Honor of Edward V. Sayre, editado por L. van Zelst, pp. 248-268. Suitland, Maryland.

Lechtman, H. 2014. Andean Metallurgy in Prehistory. En Archaeometallurgy in Global Perspective Methods and Syntheses, editado por B. Roberts y C. Thornton, pp. 361-422. Springer, New York.

Lechtman, H. y A. Macfarlane 2005. Metalurgia del bronce en los Andes Sur Centrales: Tiwanaku y San Pedro de Atacama. Estudios Atacameños 30:7-27.

Lechtman, H. y A. Macfarlane 2006. Bronce y redes de intercambio andino durante el Horizonte Medio: Tiwanaku y San Pedro de Atacama. En Esferas de Interacción Prehistóricas y Fronteras Nacionales Modernas: los Andes Sur Centrales, editado por H. Lechtman, pp. 503-550. IEP, Lima.

Le Paige, G. 1972-1973. Tres cementerios indígenas en San Pedro de Atacama y Toconao. Actas VI Congreso Nacional de Arqueología Chilena, pp.163-187. Universidad de Chile, Santiago.

Llagostera, A. 1996. San Pedro de Atacama: Nodo de complementariedad reticular. En La Integración Surandina 
Cinco Siglos Después, editado por X. Albo, M. I. Arratia, J. Hidalgo, L. Núñez, A. Llagostera, M. I. Remy y B. Revesz, pp. 17-42. Centro de Estudios Regionales Andinos Bartolomé de las Casas y UCN, Cusco-Antofagasta.

Llagostera, A. 2006. San Pedro de Atacama y el sistema reticular de interacción puneña. En Esferas de Interacción Prehistóricas y Fronteras Nacionales Modernas: los Andes Sur Centrales, editado por H. Lechtman, pp. 303-323. IEP-IAR, Lima.

Macfarlane, A. y H. Lechtman 2015. Andean ores, bronze artifacts, and lead isotopes: Constraints on metal sources in their geological context. Journal of Archaeological Method and Theory 23 (1):1-72.

Maldonado, B., T. Rehren, E. Pernika, L. Núñez y A. Leibbrant 2010. Early Cooper metallurgy in Northern Chile. En Metalla, Archäometry un Denkmalpflege, editado por O. Hahn, A. Hauptmann, D. Modarressi-Tehrani y M. Prange, pp. 96-98. Museum Bochum, Bochum.

Maldonado, B., T. Rehren, E. Pernicka, L. Núñez y A. Leibbrandt 2013. Early copper metallurgy in Northern Chile. Open Journal of Archaeometry 1 (1):1-6.

Mayer, E. 1994. Armas y herramientas de metal prehispánicas en Bolivia. En Materialien zur Allgemeinen und Vergleichende Archaologie, Band 53, Verlag, München.

Mayer, E. 1998. Armas y herramientas de metal prehispánicas en Perú. En Materialien zur Allgemeinen und Vergleichenden Archaelogie, Band 55 Verlag, München.

Money, M. 1991. El "Tesoro de San Sebastián": una tumba importante de la cultura Tiwanaku. KAVA 11:189-198.

Nielsen, A. 2007a. Bajo el hechizo de los emblemas: Políticas corporativas y tráfico interregional en los Andes Circumpuneños. En Producción y Circulación Prehispánicas de Bienes en el Sur Andino, compilado por A. Nielsen, M. Rivolta, V. Seldes, M. Vásquez y P. Mercolli, pp. 393-412. Editorial Brujas, Córdoba.

Nielsen, A. 2007b. El Período de Desarrollos Regionales en la Quebrada de Humahuaca: aspectos cronológicos. En Sociedades Precolombinas Surandinas, editado por V. Williams, B. Ventura, A. Callegari y H. Yacobaccio, pp. 235-250. Artes Gráficas Buschi S.A., Buenos Aires.

Núñez, L.1987. Tráfico de metales en el área centro-sur andina: factos y expectativas. Cuadernos del Instituto Nacional de Antropología 12:73-105.

Núñez, L. 2006. La orientación minero metalúrgica en la producción atacameña y sus relaciones fronterizas. En Esferas de Interacción Prehistóricas y Fronteras Nacionales Modernas: los Andes Sur Centrales, editado por H. Lechtman, pp. 205-260. IEP, Lima.

Núñez, L. y T. Dillehay 1995 [1979]. Movilidad Giratoria, Armonía Social y Desarrollo En Los Andes Meridionales: Patrones de Tráfico e Interacción Económica. Universidad Católica del Norte, Antofagasta.

Pifferetti, A. 1999. Arqueometalurgia de Condorhuasi-Alamito. Actas del XII Congreso Nacional de Arqueología Argentina, pp. 129-141, La Plata.

Plaza, M.T., M. Martinón-Torres y V. Figueroa 2015. Gold and silver for the Lords. Precious metal work in San Pedro de Atacama, Northern Chile, during the Middle Horizon. Trabajo presentado en Archaeometallurgy in Europe, Madrid.

Richardin, P., C. Lavier, H. Horta, V. Figueroa y N. Lira 2015 Radiocarbon dating of Atacama (Chile) snuff trays: an update on stylistic ands chronological correlations. Radiocarbon 57(5):775-784.

Rolandi, D. 1974. Un hallazgo de objetos metálicos en el área del río Doncellas (provincia de Jujuy). Relaciones de la Sociedad Argentina de Antropología VIII:153-160.
Salazar, D., H. Salinas, V. McRostie, R. Labarca y G. Vega 2010. Cerro Turquesa: Diez siglos de producción minera en el extremo norte de Chile. Actas XVII Congreso Nacional de Arqueología Chilena, pp.1085-1097. Valdivia.

Salazar, D., V. Figueroa, D. Morata, B. Mille, G. Manríquez y A. Cifuentes 2011. Metalurgia en San Pedro de Atacama durante el Período Medio: Nuevos Datos, Nuevas Preguntas. Revista Chilena de Antropología 23:123-148.

Salazar, D., H. Niemeyer, H. Horta, V. Figueroa y G. Manríquez 2014. Interaction, social identity, agency and change during Middle Horizon San Pedro de Atacama (northern Chile): A multidimensional and interdisciplinary perspective. Journal of Anthropological Archaeology 35 (1):135-152.

Stovel, E. 2013. Prehistoric atacameño ceramic styles and chronology reassessed. Chungara Revista de Antropología Chilena 45 (3):371-385.

Tamblay, J. 2004. El Cementerio Larache, los metales y la estratificación social durante el Horizonte Tiwanaku en San Pedro de Atacama. En Tiwanaku. Aproximaciones a sus Contextos Históricos y Sociales, compilado por M. Rivera y A. Kolata, pp. 31-66. Universidad Bolivariana, Santiago.

Tarragó, M. 1977. Relaciones prehispánicas entre San Pedro de Atacama (Norte de Chile) y regiones aledañas: la quebrada de Humahuaca. Estudios Atacameños 5:50-63.

Tarragó, M. 1989. Contribución al Conocimiento Arqueológico de las Poblaciones de los Oasis de San Pedro de Atacama en Relación con los Otros Pueblos Puneños, en Especial el Sector Septentrional del Valle Calchaquí. Tesis para optar al título de Doctor en Historia, Facultad de Humanidades y Artes. Universidad Nacional de Rosario, Rosario.

Tarragó, M. 1994. Intercambio entre Atacama y el borde de puna. En Taller de Costa a Selva, editado por M.E. Albeck, pp.199-213. Instituto Interdisciplinario Tilcara, UBA, Jujuy.

Tarragó, M. 1999. Las sociedades del sudeste andino. En Historia General de América Latina, editado por Unesco/Trotta, Madrid.

Tarragó, M., L. González, G. Ávalos y M. Lamamí 2010. Oro de los señores. La Tumba 11 de la Isla de Tilcara. Boletín del Museo Chileno de Arte Precolombino 15 (2):47-63.

Téllez, F. y M. Murphy 2007. El cementerio “Casa Parroquial”; un rescate afortunado. San Pedro de Atacama, Chile. En Metalurgia en la América Antigua. Teoría, Arqueología, Simbología y Tecnología de los Metales Prehispánicos, editado por R. Lleras, pp. 53-82. Fundación de Investigaciones Arqueológicas Nacionales, Banco de la República e IFEA, Bogotá.

Torres-Rouff, C. y M. Hubbe 2013. The sequence of human occupation in the atacama oases, Chile: a radiocarbon chronology based on human skeletal remains. Latin American Antiquity 24 (3):330-344.

Uribe, M. y C. Agüero 2001. Alfarería, textiles y la integración del Norte Grande de Chile a Tiwanaku. Boletín de Arqueología PUCP 5:397-426.

Uribe, M., F. Santana-Sagredo, A. Maturana, S. Flores y C. Agüero 2016. San Pedro de Atacama y La cuestión Tiwanaku en el Norte de Chile: Impresiones a partir de un clásico estudio cerámico y la evidencia bioarqueológica actual (400-1.000 d.C). Chungara Revista de Antropología Chilena 48 (2):173-198.

Varela, H. y J.A. Cocilovo 2011. Divergencia fenotípica en los Oasis de San Pedro de Atacama, Norte de Chile. Estudios Atacameños 4:101-112. 
Westfall, C. y C. González 2010. Mina Las Turquesas: un asentamiento minero lapidario preincaico en el extremo meridional circumpuneño. Actas del XVII Congreso Nacional de Arqueología, pp. 1073-1084. Ediciones Kultrún, Valdivia.

\section{Notas}

${ }^{1}$ Corresponde a único ejemplar proveniente de Solor-3, el cual no cuenta con referentes publicados. Su cuerpo es rectangular alargado con huellas de enmangue en el extremo proximal y filo irregular.

${ }^{2}$ En Tiwanaku solo se registra un fragmento metálico indeterminado procedente del sitio La K'araña (fase IV-V) (Lechtman 2003a). En el NOA se registran dos objetos correspondientes a una placacuchillo y una pinza procedentes del sitio La Rinconada (período Medio) (Gordillo y Buono 2007).

${ }^{3}$ Lamentablemente, no se cuenta con mayores antecedentes respecto a esta tumba (Llagostera, comunicación personal 2013).
${ }^{4}$ Elemento metálico esferoidal de pequeñas dimensiones, resultante del proceso de fundición.

${ }^{5} \mathrm{Se}$ ha indicado $\mathrm{Cu}$-As para objetos de contextos inciertos: una maza (Toconao) (Maldonado et al. 2010), un hacha T (Solor-3) y un hacha simple (Séquitor) (Lechtman y Macfarlane 2005, 2006).

${ }^{6}$ Correspondiente al territorio ocupado por las actuales regiones de Atacama y Coquimbo.

${ }^{7}$ A partir de observación macroscópica se observa una manufactura irregular y asimetría, la que podría estar dando cuenta de un proceso exploratorio en la elaboración de objetos más complejos como hachas y mazas. 